\title{
Multibody Dynamics Simulation of ROV Manipulator Designed for Student Competition
}

\author{
Benjamin Øygarden, Mathias Bruset and Hirpa G. Lemu* \\ Department of Mechanical and Structural Engineering and Materials Science \\ University of Stavanger \\ Stavanger N-4036, Norway \\ *Contact author: Hirpa.g.lemu@uis.no
}

\begin{abstract}
Problem-based learning or competition driven learning is nowadays used by many universities and colleges as a key tool to motivate students in higher education. In the mechanical engineering discipline, for instance, Formula Student competition, annually organized by Institution of Mechanical Engineers (IMechE) in United Kingdom, challenges student teams from around the globe to design, build and compete with a singleseat racing car. Similarly, Marine Advanced Technology Education (MATE) Center in USA organizes annual competitions to inspire students for marine education such as science, technology, engineering and mathematics (referred to as STEM). University and High School students worldwide participate in this competition with a Remotely Operated Vehicle (ROV), or underwater robots, they designed and built. In this competition, MATE acts as a client and student teams simulate a company that delivers a product in accordance with the client's stated functional requirements. In addition to the functional requirements or stated missions, the competition involves innovation in design, technical documentation, presentation, safety and marketing. In this paper, the kinematic analysis and multibody simulation of the manipulator of the ROV designed and constructed for the 2015 MATE competition that took place at St. John's in Canada is presented. The designed manipulator's kinematics while conducting the missions are studied and the motions and related loads are simulated using the multibody dynamics simulation tool ADAMS.
\end{abstract}

Keywords-multibody dynamics simulation; remotely operated vehicle; manipulator design; kinematics analysis

\section{INTRODUCTION}

Today, many technical colleges and universities conduct a number of multidisciplinary student projects aimed to attract students for science, technology, engineering, and mathematics (referred to as STEM) related education, to motivate them for learning and inspire for innovation. Based on the project/problem based learning (PBL) philosophy, most of the projects target participation in a regional, national or international competitions. This enables them to learn the engineering profession in practice, most of it much beyond what is covered by the regular teaching or curriculum. The competition spirit becomes the drive for learning by implementing the "Learn - to - win" concept. In other words, though designing and building the competition item is a short term goal for the student team's efforts, the strategic goal is stimulating the learning environment through doing. It can be referred to as competition-driven learning.
Among several international student competitions in engineering field, we find Formula Student and ROV competitions directly relevant for mechanical engineering students. In line with the goals of the MATE competition and to elevate the level of interest of students for STEM in general and subsea and marine technology in particular, student teams at University of Stavanger established UiS Subsea team in the academic year 2013/2014. The precondition for participation in the competition is that the team develop an ROV concept, design and construct the concept/the machine that can perform the tasks defined by MATE competition rules [1].

Based on the machine's size, the sensors and the tool capacity, ROVs are categorized as micro, mini, general work class and heavy work classes [2]. The micro and mini versions are lightweight machines up to $3 \mathrm{~kg}$ and $15 \mathrm{~kg}$ respectively and mostly deployed for underwater observation purposes for locations inaccessible for human divers. These vehicles are equipped with video cameras, light systems, thrustrers and few light duty sensors. The work class categories are additionally equipped with diverse capacity manipulators and sensors. They have both larger size and capacity (100 hp to $150 \mathrm{hp}$ ) and operate at water depths varying from $1 \mathrm{~km}$ to $3.5 \mathrm{~km}$.

Observing the future demand for subsea inspection, maintenance and repair operations without cabled communication, nowadays there seems large research focus on improving navigation, guidance and control of autonomous underwater vehicles (AUV) [3]. Regardless of the development efforts, the state of the art technology indicates that the subsea operations still depend on deployment of ROVs that are driven and operated by a power supplied through cables connected to a control unit on the surface, i.e. outside the water or on a floating unit such as boat/ship. Thus, the data communication of the activities done under water are visualized by information collected by video cameras, sonar and other sensors mounted on the ROV and controlled remotely by the pilot.

Within the subsea and offshore industry, many ROVs are designed and built to accomplish diverse tasks, particularly for repair and inspection works at deep waters that are unsafe for human divers [4]. Review of the literature also shows that research and development of ROV technologies is necessitated with the focus of oil and gas industries to deep waters and operations in extreme conditions [5]. This is because, though oil and gas exploration and production in shallow waters has been reached by professional divers, this is no longer an option when offshore production sites move further into deep waters. 
Most ROVs are equipped with buoyancy units that provide sufficient lift force and vehicle stability, and a number of thrusters/impellers provide manipulation of the movement of the whole unit. While the above-mentioned units of the ROV, i.e. data collection, buoyancy and thruster units guarantee the stable functionality of the vehicle, the effective accomplishment of specific tasks highly depend on the effective design of the manipulator unit. The manipulator represents the most important part of the work class vehicles and is designed in terms of diverse design specifications such as minimum outreach, lifting capacity and wrist torque.

As mentioned above, the manipulator of an ROV and AUV represents the most crucial component of the system. The underwater tasks such as picking objects and manipulating mechanical parts or turning handles to close or open valves, for instance, cannot be conducted without an appropriately designed and regulated manipulator. Thus, particular focus has been given to the design of the manipulator reported in this paper. The paper first summarizes the competition environment in Section II and presents the design considerations and analysis conducted for the main competition missions in Section III. Section IV discusses the performed multibody dynamics simulation and finally the conclusions are presented in Section V.

\section{THE COMPETITION ENVIRONMENT ORGANIZED BY MATE}

The team from University of Stavanger, UiS Subsea, participated on MATE competition for the first time in 2014, which took place at Bay National Marine Sanctuary in Thunder Alpena, Michigan, USA. On this $13^{\text {th }}$ International Student ROV Competition, 60 teams participated representing 13 different countries [6]. The main mission of this year's competition was aimed to exploring unknown shipwreck, collecting scientific samples and conservation of national marine sanctuaries using an ROV built by each student team.

The $14^{\text {th }}$ ROV competition took place (at Marine Institute of Memorial University and the National Research Council's Ocean facility) in St. John's, Canada, and 63 teams participated representing 16 different countries. The location of the competition, St. John's that is located about $2100 \mathrm{~km}$ from the polar circle [1], implies the necessity of exploring the role of ROVs in cold climate and accordingly designing the vehicle for both polar science research and the offshore oil and gas industry. The designed and built ROVs of the UiS Subsea for the first two years (2014 and 2015) are depicted in Fig. 1.

The design specifications of the vehicle were defined by the competition regulations to perform the following tasks:

- Science under the ice - such as counting species and sampling organisms, deploying an instrument, and collecting data about an iceberg;

- Subsea pipeline inspection - including replacement of a corroded section of oil pipeline and preparing a wellhead for delivery of a Christmas tree; and

- Offshore oilfield production and maintenance - such as testing the grounding of anodes on an oil platform framework, measuring the height of a wellhead, and controlling the flow of oil through a pipeline.

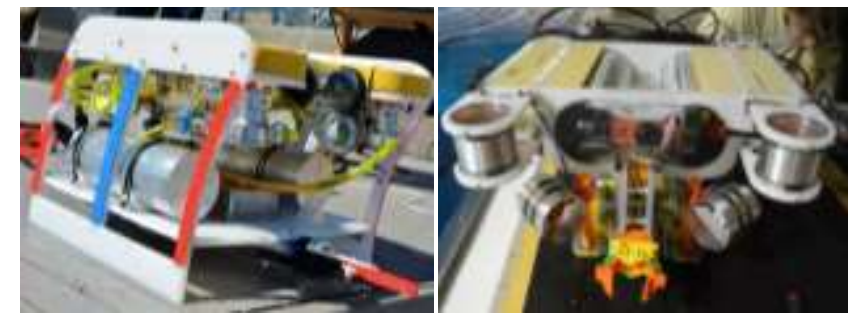

Fig. 1. Design 2014 (left) and "Thor" 2015 (right)

Recognizing the fact that the major part of the above-listed tasks is conducted by the manipulator, which simulates the actions of a human arm, the manipulator performance is extremely important for the team's competitive leverage. The work reported in this article is intended to deliver this competitiveness and hence focuses on design, kinematic analysis and multibody simulation of the manipulator.

\section{MANIPULATOR DESIGN AND ANALYSIS FOR THE MISSION}

\section{A. The manipulator design process}

Robotic manipulators designed and deployed for underwater manipulations are mostly equipped with motor or hydraulic controlled on/off type jaws and rotary wrist. These manipulators are claimed to lack sensitivity and force feedback to handle delicate and fragile objects which may lead to deformation of the manipulated objects causing expensive delays in important operations $[7,8]$. This implies that design of the manipulator and the choice of the degree of freedom (DOF) is influenced by the mission to be accomplished and the type of object to be handled by the manipulator. Based on the stated mission of the MATE competition of 2015, it was found necessary to design the manipulator with 5 degrees of freedom. These DOF are considered to be sufficient to enable the manipulator perform effectively and make its functionality flexible. In addition, the designed manipulator should be able to handle algae samples without causing damage.

As any design process, this design work involved several iterative decision-making steps on selection of, for instance, suitable forms, materials, system configurations and drives. Alternative solutions were generated and evaluated. Particular focus in terms of criticality for the operation was on mechanism of transmitting force and motion of the manipulator for the diverse tasks specified in the mission. Among the suggested alternatives are to target on rotary motion based solution (such as gear or belt drives) and translator motion based solutions (such as hydraulic, magnetic or electric pistons). The feasible alternatives are then evaluated against the performance parameters and design constraints such as leak proof operation, relative motion of parts in the mechanism, effect of mass center of the manipulator on the stability of the ROV and on the buoyancy, etc. For instance, hydraulic system based mechanism was dropped from the alternatives because it was found that the result is maintenance demanding and includes leakage related uncertainties. Fig. 2 shows some of the alternative concepts put forward in the brainstorming process at the early phase of the design process. 

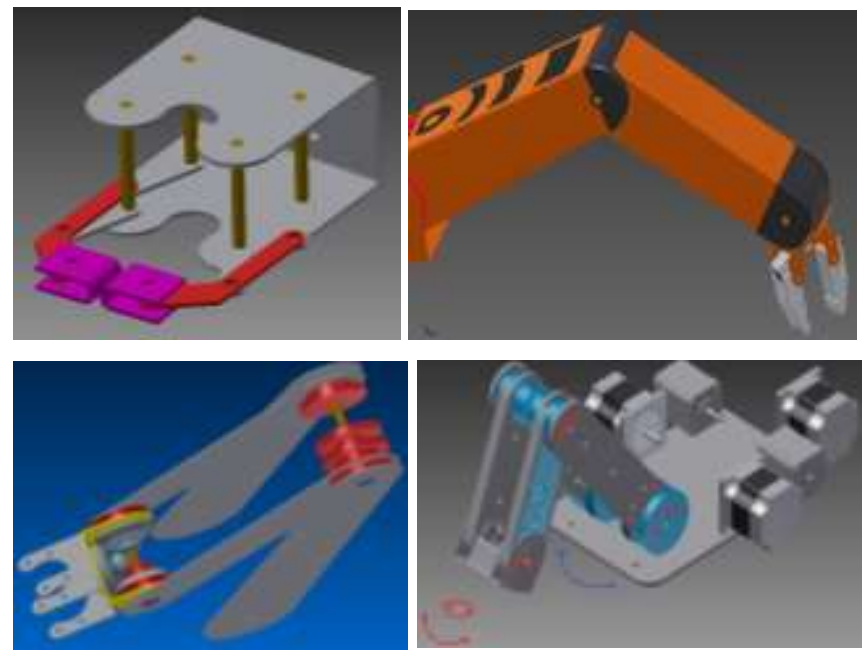

Fig. 2. Alternative design concpets at early design phase

\section{B. ROV deployment for science under ice}

For this part of the competition, for instance, one of the tasks of the manipulator was to collect samples of algae from the underside of the ice. This task is in a way challenging because of many influencing factors such as the buoyancy force, movement of water and the accuracy of the gripping force. It was simulated by letting the ROV/manipulator to pick at least 10 tennis balls and locate them under the floating ice without any damage. In addition to the fact that the balls are undamaged, having a good control of the balls and locating at the designated destination are the important measures of mission accomplishment in this part of the competition. This mission is purely dependent on the performance of the manipulator.

Fig. 3 illustrates how the manipulator is designed to have a good gripe of a ball that simulates algae sample. The forces acting on the ball when the manipulator is in action are also indicated. As illustrated in the right hand side picture, the manipulator should provide sufficient normal force that balances the effects of both gravitation and buoyancy forces acting simultaneously. According to the well established Archimedes' Principle, which states that the buoyant force on a submerged object is equal to the weight of the fluid that is displaced by the object, performance of aerodynamic machines depend on the lift provided by the buoyancy force $\left(F_{B}\right)$. This force can be calculated from

$$
F_{B}=-\rho V g
$$

where $\rho$ is the mass density of water, $V$ is the volume of the displaced water and $g$ stands for the gravitational acceleration. Accounting for the buoyancy and gravitational forces $\left(F_{g}\right)$, the total force $\left(F_{T}\right)$ acting on the ball is expressed as

$$
F_{T}=F_{g}+F_{B}=g(m-\rho V)
$$

where $m$ and $\rho V$ represent the mass of the tennis ball the displaced water respectively. To accomplish the mission, the manipulator should have a proper grip of the ball and provide sufficient frictional resistance that balance the total force given in Eq. (2).
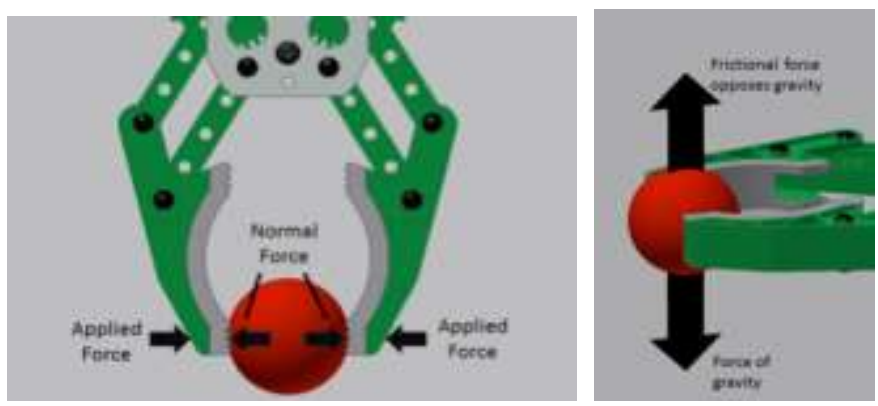

Fig. 3. Gripping of tennis ball in manipulator with acting forces

\section{Deformation test of tennis balls}

One of the competition missions are about science under ice. In this mission, tasks such as collecting algae samples and sea urchin from the seafloor, identifying and counting species of sea star, deploying acoustic sensors in a designated area and locating and measuring of iceberg are involved. The algae sample collection is simulated by tennis balls where the ROV holds a tennis ball floating under the ice and takes them to the water floor or designated position without damage or plastic deformation. This demands to test the action of the manipulator's gripper, i.e. if there exists sufficient gripping force to hold the ball and that the applied maximum force does not result in permanent deformation. The gripping capacity (existence of sufficient friction) was tested considering the effect of both gravitation and buoyancy forces acting simultaneously.

For this purpose, 6 balls were compression tested using a Universal Tensile test machine (Fig. 4). The tests were conducted stepwise at 70, 90, 100, 112, 728 and 767 N. As shown in Fig. 5, the balls loaded up to $100 \mathrm{~N}$ got their original form when unloaded (though deformed to $20 \%$ of original size and form) while those loaded by $112 \mathrm{~N}$ and over got permanent deformation. Thus, it has been concluded that the balls can be loaded with a compressive force of up to $100 \mathrm{~N}$ with certain uncertainty. Based on this result, it was decided to use $30 \mathrm{~N}$ force in the ADAMS simulation (reported in Section IV).

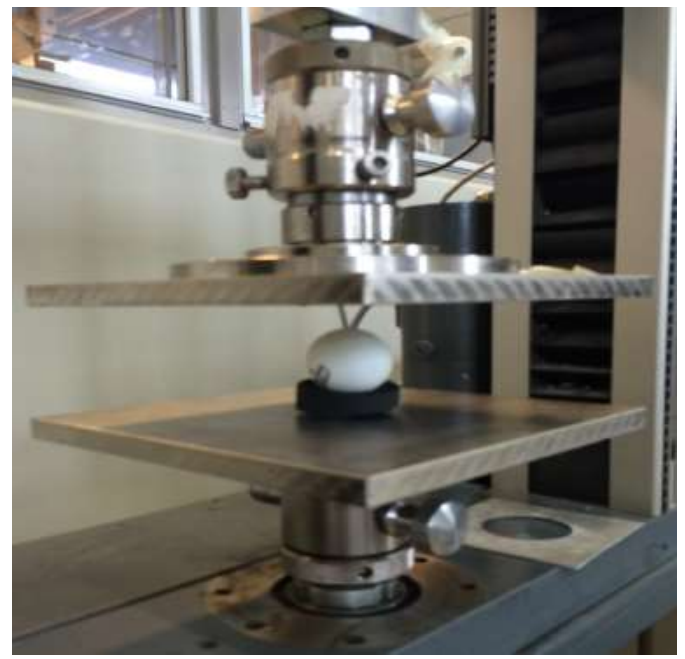

Fig. 4. Test steup in the universal test machine 


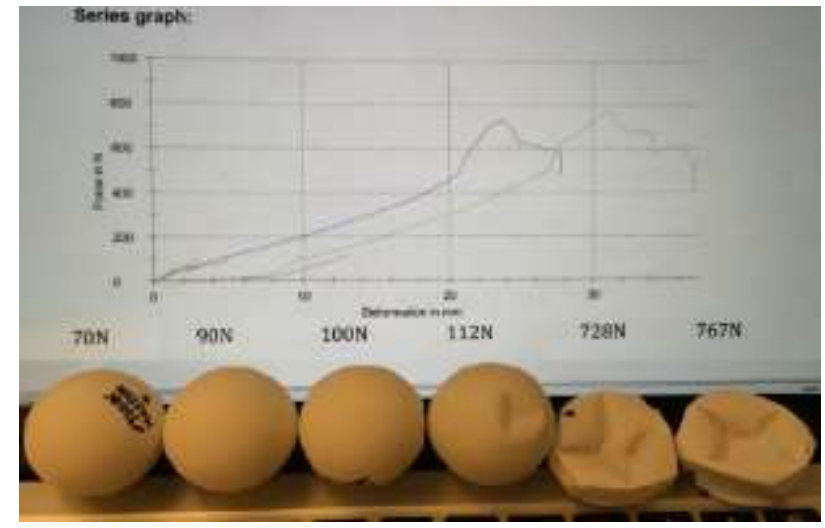

Fig. 5. Test results on universal tes machine

\section{Kinematics of the manipulator arms}

The manipulator is constructed from 3 connected links. The first link (link $\mathrm{AB}$ ) rotates the whole manipulator with respect to the ROV. Link BC is connected to link $\mathrm{AB}$ at joint $\mathrm{B}$ and is commonly called the "knee link" and it serves as a rotation link for the gripper regardless of the position of the ROV. The third link is the gripper that the manipulator uses to touch, grip and move objects.

As mechanisms, the links have relative motions with respect to each other and hence the position, velocity and acceleration of one link impacts certain motion on the rest. Calculated relative motion values assist in determination of many other key parameters for the motion of the manipulator including the forces and moments that the manipulator is exposed to while in action.

\section{E. Kinematics involved in collecting algae samples under ice}

To simulate the motion and make kinematics analysis, the manipulator arm was modelled in ADAMS software in such a way that it moves from a position where the arm indicates upwards to a position where it picks the ball "under the ice". It then locates and leaves the ball under the ice surface leaving the ball for the buoyancy force to locate it. When completed its task, the arm goes back to its upright position. Fig. 6 shows the model of the manipulator in action, at different steps of collecting the ball (simulated algae samples).

\section{MULTIBODY DYNAMICS SIMULATION OF THE MANIPULATOR}

The manipulator dynamic motions and related loads are simulated using the ADAMS software. The use of ADAMS seems intuitive specially for those accustomed to work with CAD and FEM (finite element method) systems because the software user interface and problem definition approaches have many common features. There exists however certain differences, for instance in how to design some elements and the level of simplification done without losing the functionality in the virtual test.

The dynamics of the manipulator makes mathematical calculations of the motion parameters at any time and position very difficult. Thus, use of simulation tools such as ADAMS, which is one of the powerful virtual engineering tools [9], is crucial in design and analysis of dynamic systems such as the manipulator motion. The position of the links in the manipulator are represented by the motion/rotation of motors. As a result, the position of the links at any fractions of a second can be obtained. In the following sections, the modelling approach and the simulation results for some of the main tasks of the manipulator in the MATE competition are presented.
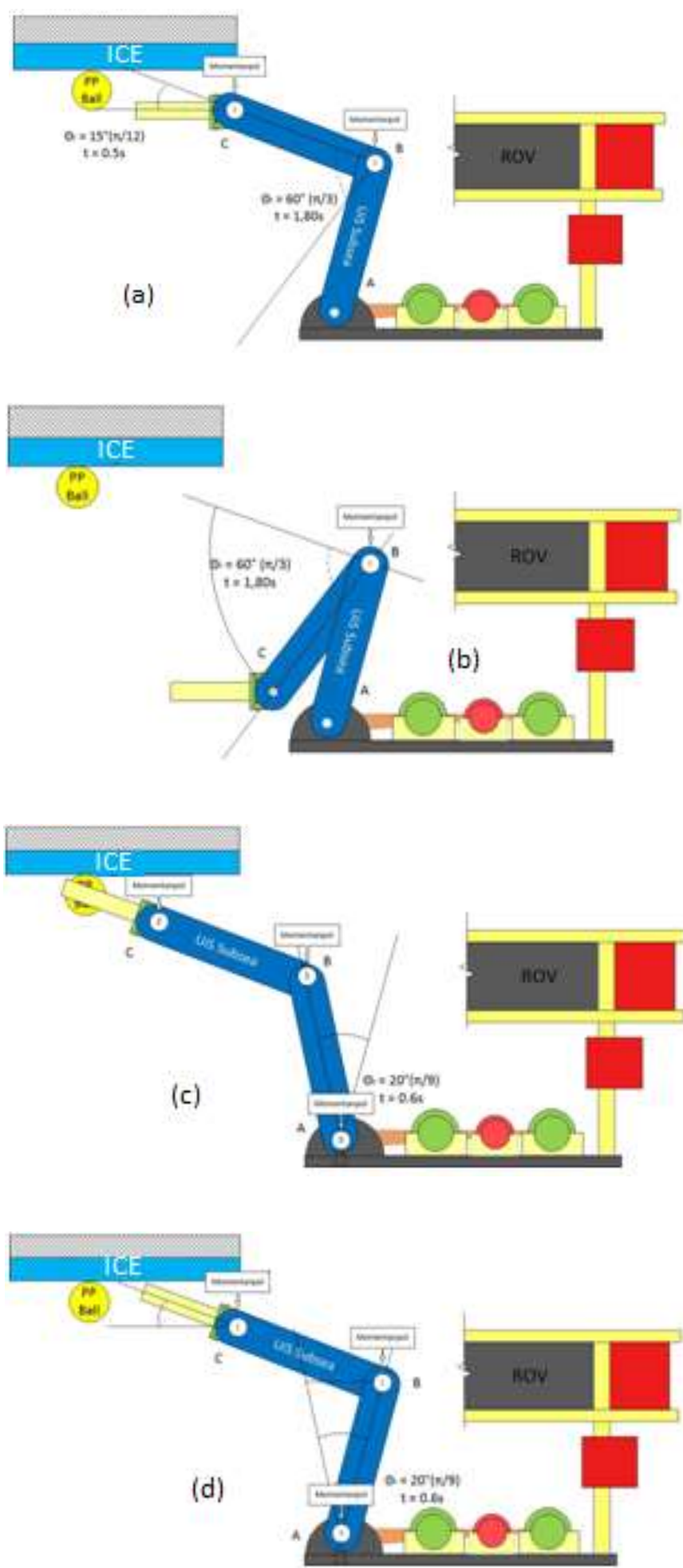

Fig. 6. Stepwise kinematics simulation of the manipulator mechanism 
A. Simulation and analysis of collecting algae samples under ice water

As part of mission 1 in the MATE competition, i.e. "Science under ice water" mission, the kinematics of the manipulator was modelled in ADAMS and simulated for 10 seconds and 50 steps. Selected positions (at $0,1,4,5,8$ and 10 seconds) of the manipulator simulations are given in Fig. 7(a) to (f) below.

Fig. 8 shows the angular position of the links as a function of time. The graphs in the figure represent the motion of the motors/links while collecting algae samples (tennis balls) under the ice is simulated.
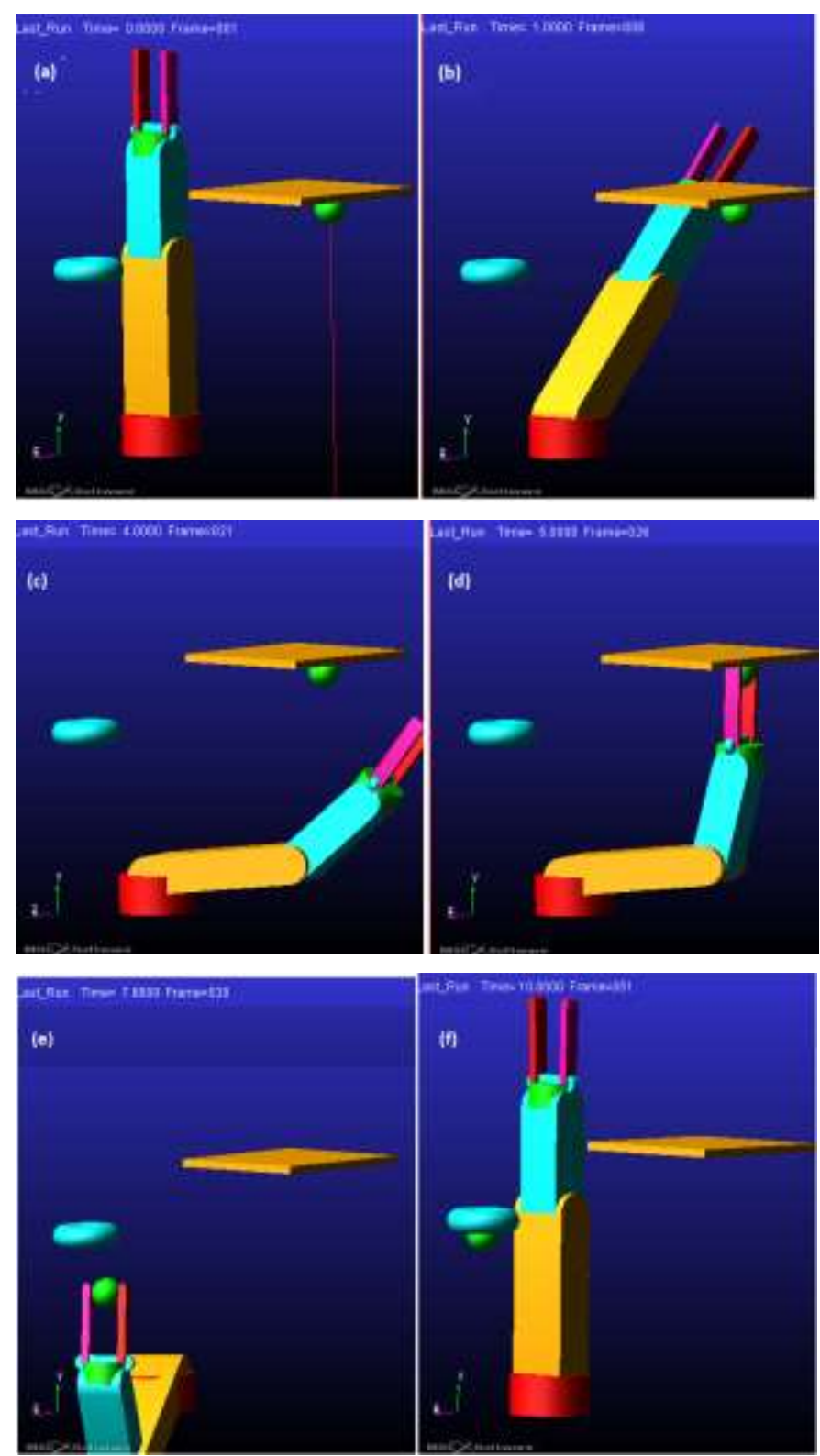

Fig. 7. Selected steps of the simulated actions of the manipulator to pick balls located under ice

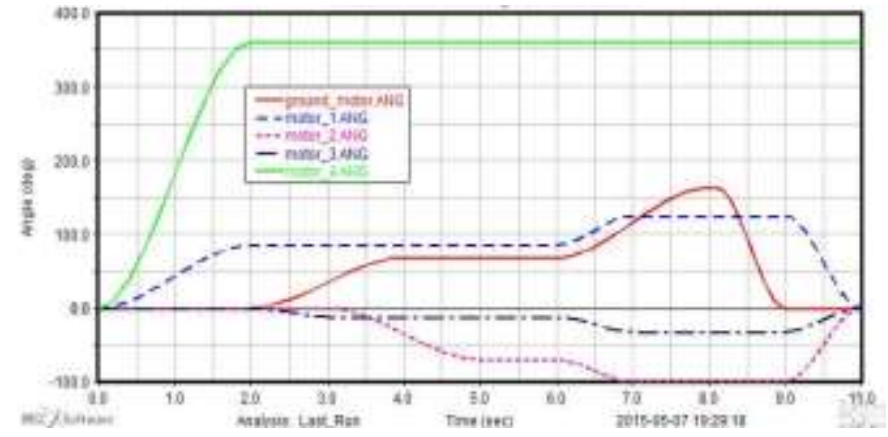

Fig. 8. Angular position of links/arms as a function of time

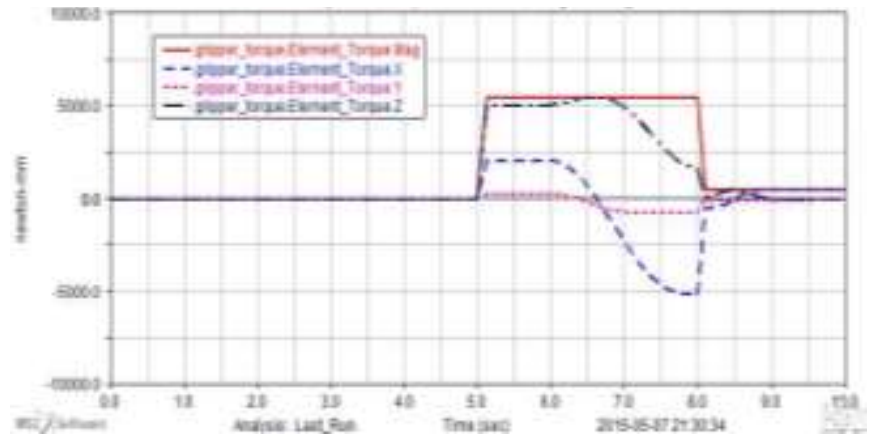

Fig. 9. Applied moment on the gripper while picking the ball

The moment applied on the manipulator is plotted in Fig. 9. This moment is made to increase from $0 \mathrm{Nm}$ to $5.5 \mathrm{Nm}$, where $1 \mathrm{Nm}$ of this moment is intended to compensate for the preloading of the torsion spring of the gripper. The plotted curves represent the moments $\mathrm{M}_{\mathrm{x}}, \mathrm{M}_{\mathrm{y}}$ and $\mathrm{M}_{\mathrm{z}}$ corresponding to the $\mathrm{x}, \mathrm{y}$ and $\mathrm{z}$ moment components respectively. The total moment (the solid line plot or designated as .gripper_torque.Element_Torque.Mag) is vector sum of these moment components.

To study how the moment (torque) at the joints varies with time, the absolute values of the moments on the manipulator arms while in motion are plotted as shown in Fig. 10. At start, the moment value showed a rapid steep rise and then dropped. This is as a result of the initial acceleration about $\mathrm{z}$-axis (motor 1). The motion in the first couple of seconds is dominated by the movement/rotation about this axis.

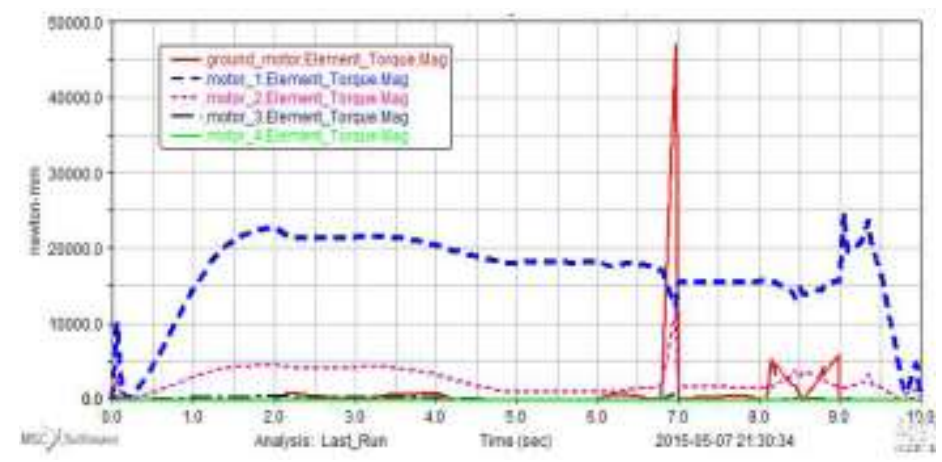

Fig. 10. Variation of torque with time on each motor 


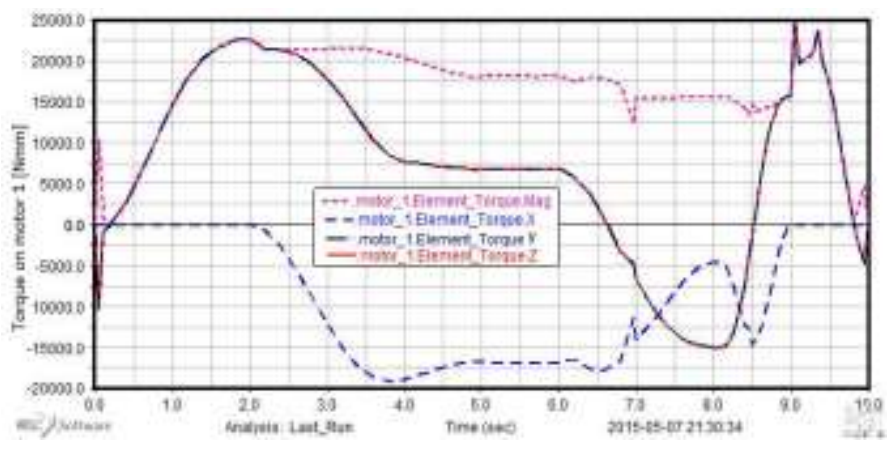

Fig. 11. Plots of moment components on motor 1

The plot of the moment components ( $\mathrm{x}, \mathrm{y}$ and $\mathrm{z}$ components) for the motion of motor 1 clearly reveals this phenomenon as shown in Fig. 11, where the plot of the absolute value of the moment and that acting about $\mathrm{z}$ axis are overlapping in the first 2 seconds. During the same time interval, the moments on motor 2 and 3 are almost identical with relatively lower slope compared with that of motor 1 . After 2 seconds, the plots drop indicating that the manipulator is decelerating to approach the grip position. The nearly horizontal plots in the time interval $4 \mathrm{~s}$ to $6.5 \mathrm{~s}$ indicate the period when the manipulator is gripping the ball. In general, the study of the simulation results in terms of the plots of the angles and moments, in this case in ADAMS, provides a more clear way to visualize the movement of each arm and their loading under action.

\section{B. Simulation and analysis for subsea pipeline inspection}

Mission 2 of the competition is directly relevant for the oil and gas industry such as underwater pipeline inspection and repair [10] as well as other underwater tasks such as marine farms [11]. To simulate this mission, the kinematics of the action of the manipulator was modelled (Fig. 12) when it attempts to open a valve mounted on a pipeline under water. This simulation is needed to test if the designed manipulator was capable of grabbing over a valve handle (with given dimensions) and is able to rotate the valve continuously. To conduct this simulation a number of simplifications were introduced including exclusion of the pipeline system and friction in the valve. As depicted in the plot of the angular displacement of the valve handle in Fig. 10 , the time 0 to $3 \mathrm{~s}$ in the motion of the manipulator is used for the initial motions of the manipulator arms, i.e. until the manipulator approaches the functional position over the valve. Then an increasing angular displacement is registered in the time interval $3-7 \mathrm{~s}$. Within this time, the valve handle rotated about 1800 degrees or $10 \pi$ radian, which corresponds to 5 rotations of the valve.

The plot in Fig. 13 is a form of STEP function used in ADAMS. A STEP function is defined by an independent value " $\mathrm{x}$ " where $\mathrm{x}_{0}$ and $\mathrm{x}_{1}$ are used to designate start and end points respectively. Correspondingly, start and end values of the function before and after the step are given by $h_{0}$ and $h_{1}$ implying that the initial value starts with $h_{0}$ until the value of " $x$ " reaches " $\mathrm{x}_{0}$ " and then changes to $\mathrm{h}_{1}$. Smooth steps are interpolated between $\mathrm{x}_{0}$ and $\mathrm{x}_{1}$ using the "best fit" values for the data set.
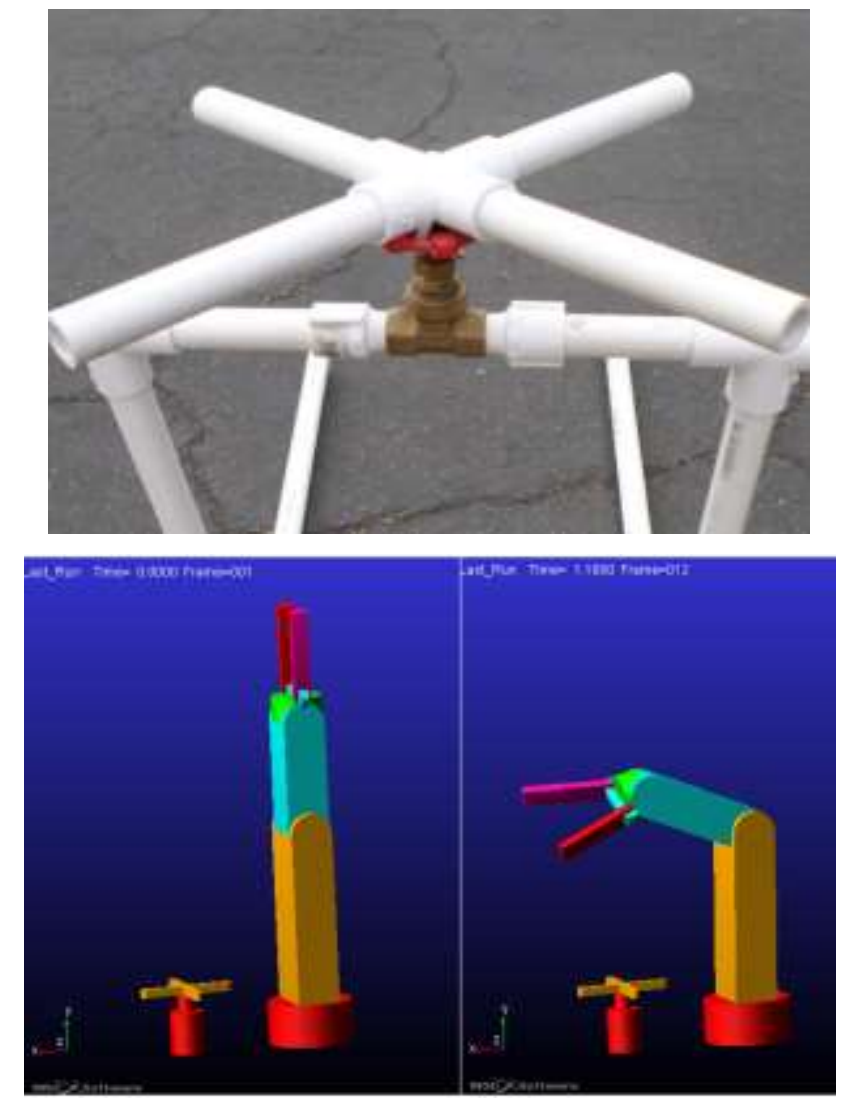

Fig. 12. The valve handle (upper) and the simulation model of manipulator (lower) for mission 2

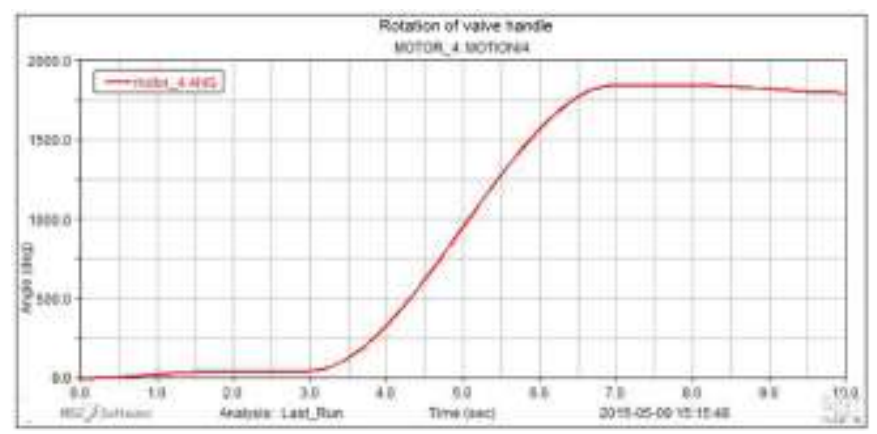

Fig. 13. Plot of angular position of valve handle with time

A typical step function is formulated as [12]

$$
S T E P=\left\{\begin{array}{cc}
h_{0} & ; x \leq x_{0} \\
h_{0}+\left(h_{1}-h_{0}\right) \cdot\left[\frac{\left(x-x_{0}\right)}{\left(x_{1}-x_{0}\right)}\right]^{2} \cdot\left[3-\left(2 \cdot\left[\frac{\left(x-x_{0}\right)}{\left(x_{1}-x_{0}\right)}\right]\right)\right] & ; x_{0}<x<x_{1} \\
h_{1} & ; x \geq x_{1}
\end{array}\right\}
$$

As can be observed from the equation (Eq. (3)), the value of $\mathrm{x}_{1}$ must not be equal to that of $\mathrm{x}_{0}$. This is because such a value results in a STEP function that has a gap and hence cannot be fitted. Using the format $\operatorname{STEP}\left(\mathrm{A}, \mathrm{x}_{0}, \mathrm{~h}_{0}, \mathrm{x}_{1}, \mathrm{~h}_{1}\right)$, where $\mathrm{A}$ represents array of $\mathrm{x}$ values, in this case time, the motion of the manipulator arms (links) for this mission are defined as given in Table I. 


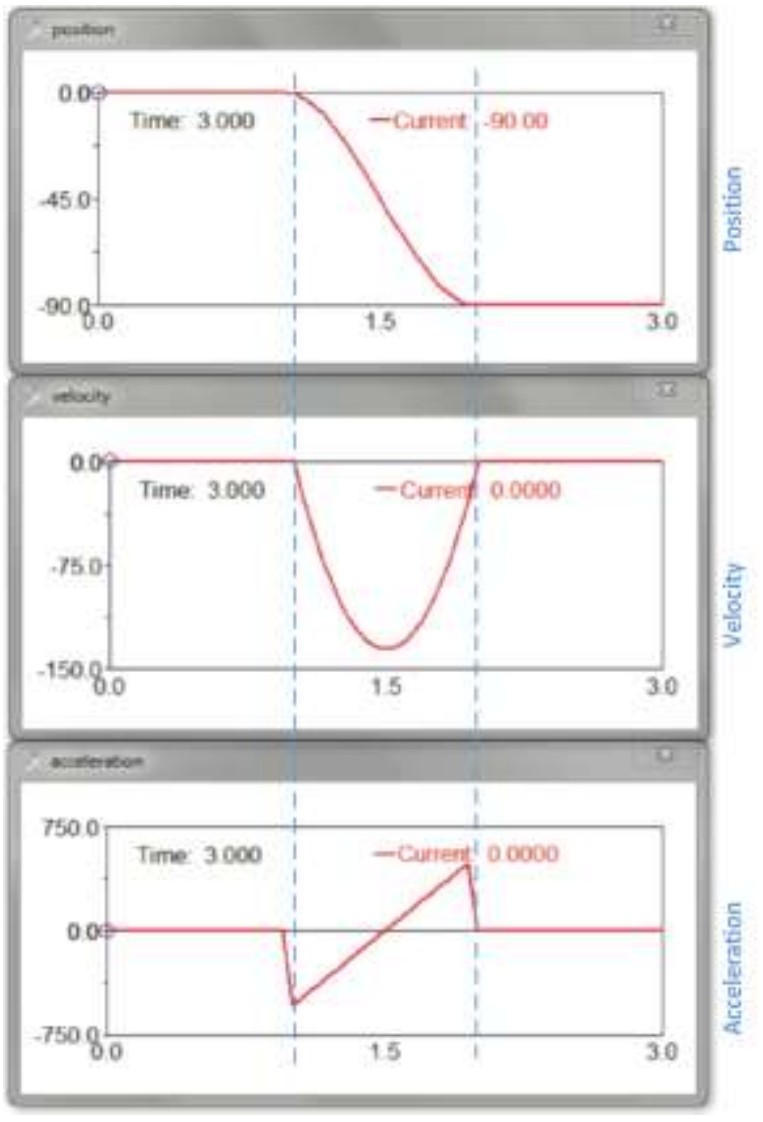

Fig. 14. Plots of the angular position defined by STEP function and the related, velocity and acceleration

TABLE I STEP-FUNCTIONS AND THE CORRESPONDING MOTIONS

\begin{tabular}{|l|l|l|}
\hline Motion & Motion function & Description \\
\hline motor_1 & step(time,0,0,2,85d) & Lowers the lower link \\
\hline motor_2 & step(time,3,0,5,-70d) & Lifts the middle link \\
\hline motor_3 & step(time, 2,0,3,-12d) & Lifts the wrist \\
\hline motor_4 & step(time,0,0,2,360d) & Rotates wrist \\
\hline ground_motor & step(time,2,0,4,68d) & Rotates the ROV \\
\hline gripper_torque & step(time,5,0,5.1,40) & Creates 40Nn force \\
\hline ground_motor & step(time,6,0,8,95d) & Rotates ROV further \\
\hline motor_1 & step(time,6,0,7,40d) & Lowes the lower link further \\
\hline motor_2 & step(time,6,0,7,-30d) & Lifts the middle link \\
\hline motor_3 & step(time,6,0,7,-20d) & Angles the wrist \\
\hline gripper_torque & step(time,8,0,8.1,-40) & Release the gripping force \\
\hline ground_motor & step(time,8.1,0,9,-163d) & Turns motor back to start \\
\hline motor_1 & step(time,9,0,10,-125d) & Turns motor 1 back to start \\
\hline motor_2 & step(time,9,0,10,100d) & Turns motor 2 back to start \\
\hline motor_3 & step(time,9,0,10,32d) & Turns motor3 back to start \\
\hline
\end{tabular}

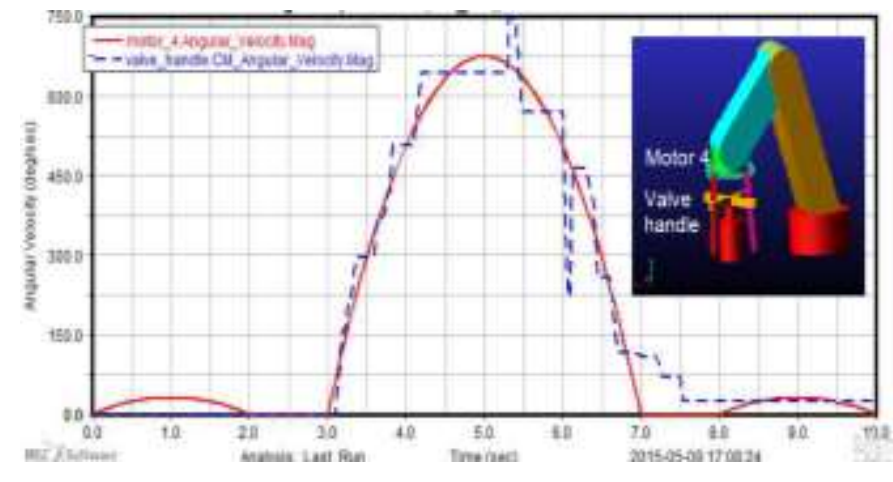

Fig. 15. Angular speed of motor 4 vs. valve handle speed

The angular speed curve of the gripper (motor) and that of the valve handle show somewhat similar trend though the angular speed of the motor is smooth, especially in the time interval $3 \mathrm{~s}$ to $7 \mathrm{~s}$, while that of the valve handle is stepped. The deviations are partly due to the lack of full contact between the gripper and valve handle surfaces and partly due to mismatch between the center of rotations. The rotation centers have an offset of $5 \mathrm{~mm}$. This type of imprecision can also take place while solving the kinematics equations of the manipulator and transforming into the desired motion of the end-effector (gripper) at finite or discrete time interval.

\section{CONCLUSION}

This paper attempted to present the research and development work done to design and simulate the manipulator of an ROV. The design and simulation of the manipulator was conducted as part of an ROV participating in an international student competition organized by MATE. The portion of the work reported in this article focuses on multibody dynamics simulation using ADAMS. Such a simulation was found important for the project because the time to make a physical prototype and to test the performance is very short. Thus, simulation or virtual prototyping avoids the need for making numerous prototypes and shorten the development process. Using limited resources, it enables testing of the manipulator's capability to execute the stated tasks in the competition manual prior to production of the parts.

The work reported in the paper is far from being complete. There still exist a number of potential improvement areas including optimization of the design of arms, the guidance and control mechanism of the manipulator and its kinematics. Furthermore, many simplifications were introduced to conducted the simulation, which may have significant impact on the accuracy of the simulation results. It is expected that a complete manipulator system may provide better accuracy. It is further recommended that some of the simulation results be verified by physical tests on a workbench prototype. 


\section{ACKNOWLEDGMENT}

This work would not be a reality without the discussions, exchange of design ideas and other contributions of Cato Sjonsti and Oddbjørn Øverland, who did a lot of excellent work producing the manipulator. The support provided by senior engineer Yaaseen A. Amith in 3D printing is also highly appreciated.

\section{REFERENCES}

[1] "MATE homepage," http://www.marinetech.org/rov-competition/ (Last accessed: 2016-06-30).

[2] NORSOK STANDARD U-102, "Remotely operated vehicle (ROV) services," http://www.standard.no/pagefiles/978/u-102r1.pdf

[3] I. Schjølberg and I. B. Utne, "Towards autonomy in ROV operations," IFAC-Conference Papers, vol. 48-2, pp. 183-188, 2015.

[4] A. Shukla and H. Karki, "Application of robotics in offshore oil and gas industry - A review Part II," Rob. Auton. Syst., vol. 75, pp. 508-524, 2016.
[5] J. Elvander and G. Hawkes, "ROVs and AUVs in support of marine renewable technologies," in: Oceans, pp. 1-6, 2012.

[6] “Ocean news and technology," https://www.oceannews.com/news/2014/06/19/2014-mate-international-rov-competition

[7] D.M. Lane, et al., "AMADEUS: advanced manipulation for deep underwater sampling," IEEE Robot. Autom. Mag. vol. 4 (4), pp. 34-45, 1997.

[8] G. Robinson, J.B.C. Davies and E. Seaton, "Mechanical design, operation and direction prediction of the AMADEUS gripper system," Int. J. Syst. Sci. vol. 29 (5), pp. 455-470, 1998.

[9] H. G. Lemu, "Virtual engineering in design and manufacturing," Advances in Manufacturing Journal, vol. 2, pp. 289 - 294, 2014.

[10] D. Mao, G. Chu, L. Yanga and Z. Lic, "Deepwater Pipeline Damage and Research on Countermeasure," Aquatic Procedia, vol. 3, pp. 180 - 190, 2015.

[11] A. R. Frost, A. P. McMaster, K. G. Saunders and S. R. Lee, "The development of a remotely operated vehicle (ROV) for aquaculture," Aquaculrural Engineering, vol. 15 (6), pp. 461-483, 1996.

[12] “ADAMS User manual," http://www.mscsoftware.com/product/adams (Last accessed: 2016.06.30). 